This is a post-peer-review, pre-copyedit version of an article published in Cellular and Molecular Neurobiology published by Springer. The final authenticated version is available online at: http://dx.doi.org/10.1007/s10571-017-0571-4 


\section{Cellular and Molecular Neurobiology \\ Effects of acute alcohol exposure on layer 5 pyramidal neurons of juvenile mice --Manuscript Draft--}

Manuscript Number:

Full Title:

Article Type:

Keywords:

Corresponding Author:
CEMN-D-17-00264R3

Effects of acute alcohol exposure on layer 5 pyramidal neurons of juvenile mice

Original Article

Ethanol; cerebral cortex; electrophysiology; pyramidal neurons; acute slice; mIPSC

Alberto Granato, M.D.

Catholic University

Milan, ITALY

Corresponding Author Secondary Information:

Corresponding Author's Institution:

Catholic University

Corresponding Author's Secondary Institution:

First Author:

Francesco Ferrini, D.V.M.

First Author Secondary Information:

Order of Authors:

Francesco Ferrini, D.V.M.

Benjamin Dering, Ph.D.

Andrea De Giorgio, Ph.D.

Laura Lossi, D.V.M.

Alberto Granato, M.D.

Order of Authors Secondary Information:

Funding Information:

Università degli Studi di Torino

Dr. Francesco Ferrini

Dr. Laura Lossi

Università Cattolica del Sacro Cuore Dr. Alberto Granato

\section{Abstract:}

Early-onset drinking during childhood or preadolescence is a serious social problem. Yet, most of the basic neurobiological research on the acute effects of ethanol has been carried out on adult or early postnatal animals. We studied the effect of alcohol exposure on the basic electrophysiological properties and cell viability of layer 5 pyramidal neurons from the somatosensory cortex of juvenile (P21-P23) C57BL/6N mice. After bath application of $50 \mathrm{mM}$ ethanol to acute slices of the somatosensory cortex, no adverse effects were detected on cells survival, whereas the input resistance and firing rate of layer 5 neurons were significantly reduced. While the effect on the input resistance was reversible, the depressing effect on cell firing remained stable after 6 minutes of alcohol exposure. Ethanol application did not result in any significant change of mIPSC frequency, amplitude, and rise time. A slight increase of mIPSC decay time was observed after 6 minutes of ethanol exposure. The molecular mechanisms leading to these alterations and their significance for the physiology of the cerebral cortex are briefly discussed.

Response to Reviewers:

Reviewer's comments and our answers are interleaved. Changes to the text are highlighted in red in the manuscript file.

\section{REVIEWER:}

The additional references provided are helpful. However, there are several canonical reviews devoted to this area explicitly that would be hugely beneficial to readers less familiar with the nuances of this research area. Dr. LP Spear et al. has authored many such reviews for example. 
Response:

As suggested by the Reviewer, the following text has been added to Introduction: "Indeed, ethanol affects different cortical regions and induces important ontogenetic alterations during adolescence, which critically influence subsequent drug selfadministration in adulthood (Spear 2016)".

\section{REVIEWER:}

I understand referencing work using very similar methods. However, it seems relevant to at least briefly discuss how the current work fits in the context of developmental patch work in other brain areas/rodents strains. For example, findings from Brodie and Appel (2000) suggest that ethanol may have very different effects as a function of age and brain region examined, as well as in other rodent strains.

\section{Response:}

To point out that different alcohol effects might be observed, depending on the rodent strain and/or developmental stage, the following text has been added to Discussion: "As a more general remark on experimental alcohol studies, it should be taken into account that different effects of ethanol can be observed not only in different brain regions, but also as a function of age and rodent strains (Brodie and Appel 2000; Ikonomidou et al. 2000; Dou et al. 2013)."

\section{REVIEWER:}

Related to this last point, this reviewer was only able to find information pertaining to the $/ 6 \mathrm{~N}$ substrain of the C7BL mice when Charles River (Italy) was queried. Differences between these 2 substrains ( $/ 6 \mathrm{~J}$ and $/ 6 \mathrm{~N}$ ) in ethanolrelated behaviors have been reported, and it seems reasonable that these may be due to differences in ethanolinduced brain function. Please confirm which of these substrains was used. Please also include details on the number of generations from the founding stock mice were that were used for experiments. Were littermates used for these studies or was there any effort to test mice from different litters to control for this potential factor?

\section{Response:}

We thank the Reviewer for raising this point. Both substrains of C57BL/6 mice were ordered in the past by the University of Turin. The ones used in the present study were purchased from Charles River Italy, as indicated in the manuscript, but they were actually $\mathrm{C} 57 \mathrm{BL} / 6 \mathrm{~N}$, not $\mathrm{C} 57 \mathrm{BL} / 6 \mathrm{~J}$, as mistakenly indicated in the previous version of the manuscript. We have now corrected the substrain indication. In addition, the phrase of Material and Methods: "All animals used were bred in house" has been modified as follows: "All animals used were bred in house for four generations. Experiments were conducted on mice obtained from different litters."

Finally, according to the instructions for authors, we have slightly modified Figure 4 (part d), in order to remove reference to colors from the caption. 


\title{
Effects of acute alcohol exposure on layer 5 pyramidal neurons of juvenile mice
}

\author{
Francesco Ferrini ${ }^{1}$, Benjamin Dering ${ }^{2}$, Andrea De Giorgio ${ }^{3}$, Laura Lossi ${ }^{1}$, Alberto Granato ${ }^{4}$
}

1. Department of Veterinary Sciences, University of Turin, Largo Braccini 2, 10095 Grugliasco, Italy.

2. Faculty of Natural Sciences, University of Stirling, Stirling, United Kingdom.

3. Faculty of Psychology, University eCampus, Novedrate, Italy.

4. Department of Psychology, Catholic University, Milan, Italy

Corresponding Author:
Alberto Granato
Department of Psychology, Catholic University
Largo A. Gemelli 1, 20123, Milan, Italy
Ph.: +3902 72348588
alberto.granato@unicatt.it 


\section{Abstract}

Early-onset drinking during childhood or preadolescence is a serious social problem. Yet, most of the basic neurobiological research on the acute effects of ethanol has been carried out on adult or early postnatal animals. We studied the effect of alcohol exposure on the basic electrophysiological properties and cell viability of layer 5 pyramidal neurons from the somatosensory cortex of juvenile (P21-P23) C57BL/6N mice. After bath application of $50 \mathrm{mM}$ ethanol to acute slices of the somatosensory cortex, no adverse effects were detected on cells survival, whereas the input resistance and firing rate of layer 5 neurons were significantly reduced. While the effect on the input resistance was reversible, the depressing effect on cell firing remained stable after 6 minutes of alcohol exposure. Ethanol application did not result in any significant change of mIPSC frequency, amplitude, and rise time. A slight increase of mIPSC decay time was observed after 6 minutes of ethanol exposure. The molecular mechanisms leading to these alterations and their significance for the physiology of the cerebral cortex are briefly discussed.

\section{Keywords}

Ethanol; cerebral cortex; electrophysiology; pyramidal neurons; acute slice; mIPSC.

\section{Author Contributions}

Francesco Ferrini designed and performed the experiments, analyzed the data and wrote the manuscript. Benjamin Dering designed and performed the experiments and analyzed the data. Andrea De Giorgio performed the experiments and analyzed the data. Laura Lossi performed the experiments and analyzed the data. Alberto Granato designed and performed the experiments, analyzed the data and wrote the manuscript. All the authors have read and approved the manuscript. 


\section{Introduction}

Alcohol abuse is one of the most serious problems faced by our society, with a tremendous impact on public health and economy (Rehm et al. 2009). The issues raised by childhood- and preadolescence-onset drinking (Donovan 2013) are even more worrisome, since early-onset drinking is positively associated with the development of alcohol dependence later in life (Hingson et al. 2006). In particular, alcohol consumption during adolescence has been associated with disruptions of many normal developmental processes, potentially disturbing the maturation of higher-order executive functions (McMurray et al. 2016). Indeed, ethanol affects different cortical regions and induces important ontogenetic alterations during adolescence, which critically influence subsequent drug self-administration in adulthood (Spear 2016). Surprisingly, despite the huge amount of work devoted to experimental research on alcohol dependence, little is known about the neurobiology of alcohol effects in juvenile lab animals.

Pyramidal neurons of layer 5 (L5), the most complex cells among those providing the output from the cerebral cortex (Ramaswamy and Markram 2015), are highly sensitive to the long-term deleterious effects of ethanol. Early exposure to alcohol during the first stages of development can permanently modify the electrophysiological properties of these neurons (Granato et al. 2012). As to the acute effects of ethanol, there are reports describing the alterations of the electrophysiological parameters of L5 neurons after alcohol application on acute slices from adult rodents (Proctor et al. 1992; Sessler et al. 1998). Other studies, instead, focused on effects of ethanol in cortical slices from postnatal rodents showing not only effects on neuronal activity, as in the adult, but also on cell survival (Sanderson et al. 2009). Conversely, little is known about the effects of ethanol on layer 5 neurons of juvenile rodents (P21-23), a developmental stage considered to correspond to "early adolescence" in humans. To address this issue and fill the gap of knowledge at such a critical age, we designed in vitro electrophysiological experiments and cell viability tests on P21-P23 C57BL/6 mice, the strain most commonly adopted in alcohol research as genetic background for the generation of transgenic mice (Heit et al. 2015).

\section{Materials and Methods}

Animals. Juvenile male (P21-P23) C57BL/6N mice were used in the present study. The breeding colony was purchased from Charles River Laboratories (Italy). All animals used were bred in house for four generations. Experiments were conducted on mice obtained from different litters. To avoid exposing mice to unwanted stressful events prior to the experimental procedures, adolescent mice were not weaned until sacrifice. 
All the experiments were conducted in accordance with the Society for Neuroscience Policies on the Use of Animals and Humans in Neuroscience Research, as well as current Italian and EU regulation on animal experimentation and welfare.

Electrophysiology. Mice were anaesthetized (Pentothal $50 \mathrm{mg} / \mathrm{Kg}$ i.p.) and, after decapitation, the brain was quickly removed. Coronal slices $(300 \mu \mathrm{m})$ of the primary somatosensory cortex were cut on a vibratome in ice-cold, oxygenated solution containing (in $\mathrm{mM}$ ): 250 sucrose, $25 \mathrm{NaHCO}_{3}, 2.5$ $\mathrm{KCl}, 1.25 \mathrm{NaH}_{2} \mathrm{PO}_{4}, 1 \mathrm{MgCl}_{2}, 25$ glucose, $2 \mathrm{CaCl}_{2}$. The composition of the ACSF used for all the subsequent procedures was the same as the cutting solution, with the exception that the sucrose was replaced by $\mathrm{NaCl} 125 \mathrm{mM}$. Slices were kept at $34^{\circ} \mathrm{C}$ in oxygenated ACSF for $1 \mathrm{~h}$ and then at room temperature until use. Recording pipettes (4-8 M $\Omega$ ) were made from borosilicate glass capillaries. Whole-cell current clamp recordings were obtained from the soma of visually identified pyramidal neurons of layer 5 using IR-DIC optics. Recordings were carried out using a Multiclamp 700B amplifier (Molecular Devices, Sunnyvale, CA) and acquired with a Digidata digitizer (Molecular Devices). Series resistance $\left(\mathrm{R}_{\mathrm{s}}\right)$ was monitored throughout the recordings and compensated using the bridge balance circuit of the amplifier. Recordings were discarded if $R_{\mathrm{s}}$ changed by more than $20 \%$ during the experimental procedure.

Current clamp recordings were obtained with an internal solution containing (in $\mathrm{mM}$ ): $135 \mathrm{~K}$ gluconate, $5 \mathrm{KCl}, 10 \mathrm{HEPES}, 2 \mathrm{MgCl}_{2}$, 4Na2 $\mathrm{ATP}, 0.4 \mathrm{NaGTP}, \mathrm{pH}$ 7.2. For some recordings, the intracellular solution contained $0.1 \%$ Lucifer Yellow. Several steps of hyperpolarizing and depolarizing current were injected into the soma (increments of $50 \mathrm{pA}$; duration: $800 \mathrm{~ms}$ ). Once stable recordings were obtained in normal ACSF (preEt), the slice was perfused with ACSF +50 $\mathrm{mM}$ ethanol and additional recordings were obtained from the same cell 3 and 6 minutes after the beginning of ethanol application ( $3 \mathrm{mEt}$ and $6 \mathrm{mEt}$, respectively). Recordings were also performed 5 and 10 minutes after the beginning of alcohol washout; cells were discarded if the number of spikes did not return to at least $40 \%$ of baseline during washout.

Active and passive membrane properties were analyzed using Igor Pro (Wavemetrics, Lake Oswego, OR). The following subthreshold parameters were measured: resting membrane potential, input resistance, membrane time constant, depolarizing voltage sag (calculated as $\left(\mathrm{V}_{\max }-\mathrm{V}_{\mathrm{ss}}\right) /$ $\mathrm{V}_{\max }$, where $\mathrm{V}_{\max }$ is the transient voltage peak reached soon after a hyperpolarizing current step and $\mathrm{V}_{\mathrm{ss}}$ is the steady state voltage). Measured suprathreshold parameters were: spike threshold, spike amplitude, spike half-width (i.e. the spike width at half amplitude), afterhyperpolarization, interspike interval (ISI), and ratio between the last and $1^{\text {st }}$ ISI of a spike train.

For voltage clamp recordings $\mathrm{K}$ gluconate and $\mathrm{KCl}$ in the intracellular solution were substituted with $\mathrm{CsCl}(140 \mathrm{mM})$. Miniature inhibitory post-synaptic currents (mIPSCs) were isolated in 
presence of TTX (1 $\mu \mathrm{M}$; Tocris Cookson, Bristol, UK), APV (40 $\mu \mathrm{M}$; Sigma, St. Louis, MO, USA), NBQX $\left(10 \mu \mathrm{M}\right.$; Sigma) at a holding potential of $-60 \mathrm{mV}$. Under these experimental conditions $\mathrm{E}_{\mathrm{Cl}}$ is near $0 \mathrm{mV}$, thus IPSCs are inwardly directed.

mIPSCs were analyzed by Mini Analysis software (Synaptosoft Inc., Decataur, GA). mIPSC frequency and amplitude were sampled for periods of $100 \mathrm{~s}$. Rise time and decay time kinetics were analyzed by averaging over 100 synaptic events/cell per each experimental condition. Rise time was defined as the duration of the rise from 10 to $90 \%$ of the peak. Decay time was calculated by fitting the $10-90 \%$ decay phase with a monoexponential function and expressed as time constant $\tau$.

Cell viability assay. After recovery in ACSF, three slices for each experimental condition (control, $3 \mathrm{mEt}$, and $6 \mathrm{mEt}$ ) were transferred into ACSF containing $1.5 \mathrm{mM}$ propidium iodide (PI) and incubated for $10 \mathrm{~min}$ at room temperature. They were then washed three times in plain ACSF, followed by 30 min fixation in $4 \%$ paraformaldehyde in $0.1 \mathrm{M}$ phosphate-buffered saline (PBS) (pH 7.4-7.6). Slices were then washed in PBS ( $2 \times 10 \mathrm{~min})$ and double distilled water $(2 \times 5 \mathrm{~min})$ and mounted in fluorescent free medium (Vectashield ${ }^{\circledR}$ Antifade Mounting Medium, Vector Laboratories, Burlingame, CA). They were then photographed using a Leica DM6000 wide-field fluorescence microscope (Leica Microsystems, Wetzlar, Germany) with a 20x lens. For each slice, 10 randomly selected microscope fields were photographed at a resolution of $1392 \times 1040$ pixels $\left(0.3084 \mathrm{~mm}^{2}\right)$ and PI stained nuclei were counted with the "Count Particles" function of the ImageJ software (NIH, Bethesda, MD, USA) in an interval of area size between 12.56 and 78.50 $\mu \mathrm{m}^{2}$ (diameter $4-10 \mu \mathrm{m}$ ). Values were expressed as number of PI stained nuclei $/ \mathrm{mm}^{2}$.

Statistical analysis. Statistical differences of all grouped electrophysiological data, but ISI, were evaluated using the ANOVA for repeated measures test followed by Bonferroni post-hoc. As ISI data distribution is not normal, differences were analyzed through the non-parametric Wilcoxon signed-rank test. Differences in the number of dead cells in the cell viability assay were tested by a one-way ANOVA. All values are expressed as mean \pm standard error of the mean (s.e.m.). Differences were considered to be significant at $P<0.05$.

\section{Results}

Acute effects of ethanol on neuronal activity in L5 pyramidal neurons were assessed after 3 minutes $(3 \mathrm{mEt})$ and 6 minutes $(6 \mathrm{mEt})$ of slice exposure to $50 \mathrm{mM}$ ethanol, a concentration previously adopted in several functional studies in vitro and in vivo (e.g.: Criswell et al. 2008; Ehlrich et al. 2012, Huang e al. 2012) and known to mimic ethanol levels in the blood of heavy drinkers (Han et al. 1998; Ehlrich et al. 2012). This brain concentration of ethanol causes cognitive dysfunction in humans and animal models, as well as altered synaptic function (reviewed in Tipps et al., 2014; 
White, 2003; Zorumski et al., 2014). Moreover, levels of ethanol in the central nervous system have been shown to match with blood levels in animal models (Gilpin et al., 2009; Smolen and Smolen, 1989).

To exclude the possibility that the effects of ethanol on neuronal activity could be linked to its proapoptotic effects (Olney, 2014) we have evaluated the incidence of cell death with PI in any of the three experimental conditions of the present study (Fig. 1). Dead cells are easily spotted as their nuclei are intensely fluorescent following PI uptake. PI stained nuclei are scattered throughout the slice and there are no obvious differences in the number of positive nuclei between the three experimental conditions (Fig. 1a-c) Fig. 1d shows the results of statistical analysis in graph form. After one-way ANOVA there are no statistically significant differences among the three experimental groups $\left(\mathrm{F}_{2,30}=0.467 ; \mathrm{P}=0.63\right)$.

All the cells recorded in current clamp and used for the quantitative evaluation $(n=8)$ were regular spiking L5 pyramidal neurons with a moderate spike frequency adaptation and input resistance ranging from 92 to $262 \mathrm{M} \Omega$ (mean \pm s.e.m. $=161.53 \pm 20.75 \mathrm{M} \Omega$ ). The morphological control performed on neurons filled with Lucifer yellow confirmed the presence of basal dendrites and of an apical dendrite reaching the most superficial layers of the cortex. Figure 2 summarizes the main subthreshold parameters before ethanol exposure and 3-6 minutes after the beginning of ethanol bath application. The mean resting membrane potential was hyperpolarized by about $5 \mathrm{mV}$ after ethanol application, with a statistically significant difference between preEt and $3 \mathrm{mEt}$, and between preEt and 6mEt (Fig. 2a). The input resistance and the membrane time constant showed a similar trend (Fig. 2b-c): they were significantly lower than preEt at 3mEt, while they returned to baseline levels at $6 \mathrm{mEt}$. No changes where observed in depolarizing voltage sag (Fig. 2d). Suprathreshold parameters obtained upon somatic injections of depolarizing current are shown in Figure 3. As demonstrated by the ISI, the firing frequency was significantly reduced at both $3 \mathrm{mEt}$ and $6 \mathrm{mEt}$, as compared to preEt (Fig. 3a. This behavior was substantially maintained for all the levels of injected current (rheobase, 50 pA, and 100 pA above rheobase; Fig. 3b-d). For current injections 50 pA above rheobase, there was a significantly higher frequency adaptation at $6 \mathrm{mEt}$, as compared to preEt (Fig. 3c - inset). Other action potential parameters (threshold, amplitude, half-width, and afterhyperpolarization; Fig. 3e-h) were not significantly different before and after ethanol exposure, except threshold values that were significantly higher at $6 \mathrm{mEt}$ than at $3 \mathrm{mEt}$.

As ethanol is known to enhance GABAergic inhibition (Lobo and Harris 2008; Förstera et al. 2016), we tested whether alterations in active and passive membrane properties were also paralleled by increased miniature inhibitory postsynaptic transmission (mIPSCs; Fig. 4). Ethanol application did not significantly alter mIPSC frequency, suggesting a lack of pre-synaptic effects (Fig. 4b). 
Also, no differences were observed in mIPSC amplitude and rise time (Fig. 4c-e). However a slight but significant increase in decay time was observed at $6 \mathrm{mEt}$ as compared to preEt level (Fig. 4f).

\section{Discussion}

The present study provides a comprehensive description of the effects of alcohol exposure on the electrophysiological properties of neocortical pyramidal neurons in juvenile rodents. The deleterious actions of ethanol in the immature brain mainly involve a direct depression of neuronal activity (Lotfullina and Khazipov 2017). The inhibitory effects of ethanol decrease with age and, in adult animals, ethanol only mildly depresses neuronal firing. In newborn P3-P9 rats, the overall suppression of neuronal activity is mediated by both the depression of NMDA receptors and the potentiation of GABAergic activity (Galindo et al. 2005, Sanderson et al., 2009). This combined effect has been associated with ethanol-induced apoptosis in the developing brain (Lotfullina and Khazipov 2017). Although the suppressive effects of ethanol on cortical activity and the consequent apoptosis decrease with age, yet in adult cortical neurons ethanol still decreases NMDA currents, spike firing, and input resistance with no effects on GABAergic transmission (Badanich et al. 2013 Sessler et al. 1998). Our data confirm that ethanol has suppressive effects on firing activity of pyramidal neurons from juvenile adolescent mice, without causing detectable cell death. Unlike adult neurons, however, it also causes a mild post-synaptic enhancement of GABAergic transmission, which may further amplify the suppressive effect on firing activity.

In particular, we have shown a significant reduction of the input resistance and membrane time constant after 3 minutes of $50 \mathrm{mM}$ ethanol bath application, accompanied by a hyperpolarization of resting membrane potential. Consistently, firing rate was also significantly reduced after both 3 and 6 minutes of alcohol exposure. Our data are in line with previous observations made on acute slices of adult rodents, although some discrepancies among different works can be found. Sessler et al. (1998) reported a decreased firing rate of L5 neurons of young (125-200 gr) rats after bath application of ethanol. A reduction in input resistance was also observed, while the authors did not find consistent changes of membrane potential (Sessler et al. 1998). Conversely, the hyperpolarization, in presence of a small, non-significant reduction of input resistance, was observed in rats by Proctor and coworkers (1992). In large regular spiking neurons of the sevenweek-old mouse orbitofrontal cortex, the application of ethanol led to a reduction of spike firing and input resistance, accompanied by hyperpolarization (Badanich et al. 2013). These effects seem to be region-specific, since in hippocampal slices the acute exposure to alcohol was more frequently accompanied by depolarization rather than hyperpolarization (Siggins et al. 1987). As a more general remark on experimental alcohol studies, it should be taken into account that different effects 
of ethanol can be observed not only in different brain regions, but also as a function of age and rodent strains (Brodie and Appel 2000; Ikonomidou et al. 2000; Dou et al. 2013).

There are several possible explanations for the decrease in intrinsic excitability and input resistance observed in the present study. The potentiation of GABAergic transmission by ethanol has been repeatedly observed in brain slices (reviewed in Weiner and Valenzuela 2006). Our data on mIPSCs, however, show no significant difference of amplitude and frequency after exposure to ethanol. These findings are in agreement with those obtained by Fleming et al. (2009) on cultured cortical neurons. In other studies, a change of miniature or spontaneous IPSCs has been observed after exposure of dissociated cortical neurons to $100 \mathrm{mM}$ ethanol (Marszalec et al. 1998; Moriguchi et al. 2007) and increased presynaptic release of GABA has been reported in different immature rodent models (Galindo et al. 2005, Sanderson et al., 2009). Even in absence of frequency/amplitude modifications, the slightly longer duration of mIPSCs observed in our study after 6 minutes of ethanol application, and therefore the increased GABA-mediated $\mathrm{Cl}^{-}$charge transfer, may partly explain the decreased excitability of pyramidal neurons. However, as no effects on decay time were detected after 3 minutes, the increased inhibitory synaptic transmission is unlikely to underlie early ethanol-induced changes in cell activity. On the other hand, we cannot exclude that ethanol may also potentiate extrasynaptic $\mathrm{GABA}_{\mathrm{A}}$ receptors, thus challenging tonic GABAergic inhibition. Indeed, ethanol-mediated effects on tonic inhibition have been previously reported in hippocampal neurons (Wei et al. 2004). Further investigations are thus required to specifically address this point in cortical neurons.

Another effect potentially accounting for a reduction of input resistance is the potentiation of hyperpolarization-activated currents $\left(\mathrm{I}_{h}\right)$. It is known that ethanol augments $\mathrm{I}_{h}$ in neuronal and nonneuronal cell types (e.g. Okamoto et al. 2006; Chen et al. 2012). The alcohol-mediated potentiation of $\mathrm{I}_{\mathrm{h}}$ on hippocampal interneurons is more effective in adolescent than in adult rats (Yan et al. 2009). However, the expression of HCN channels mediating $I_{h}$ at somatic locations of L5 pyramidal neurons is constantly low during development (Atkinson and Williams 2009). Furthermore, in the present study we did not observe a significant change of the depolarizing sag after injection of hyperpolarizing current. Therefore, the effect of ethanol on $\mathrm{I}_{\mathrm{h}}$, if any, is unlikely to be the main factor responsible for the decrease of input resistance. We cannot rule out that ethanol can affect other ion channels, as the electrophysiological properties of neurons are the result of a combined effect of several conductances (e.g., Day et al. 2005).

Interestingly, we have also found that some of the ethanol-related changes are time-dependent. In fact, while the decreased spike firing is maintained throughout the alcohol superfusion, the input resistance and membrane time constant display a more complex time course, showing a significant 
decrease after 3 minutes of alcohol application, followed by a return to control values after 6 minutes. This biphasic action of ethanol might involve slow-onset processes such as ion channel phosphorylation (reviewed in Trudell et al., 2014).

Whatever the mechanism accounting for the alterations observed in the present study, they can impair the function of juvenile neurons acutely exposed to alcohol. The changes of membrane time constant, besides affecting synaptic integration, can also modify the induction of spike-timing dependent plasticity (Fuenzalida et al. 2007). The reduced intrinsic excitability of pyramidal neurons can have deep consequences on network activity and on plastic adjustments required for learning and memory processes (see Cohen et al. 2017, for a discussion on the interplay among neuron excitability, plasticity, and network remodeling). These alterations are expected to have a dramatic effect on cortical activity of young drinkers, since the blood ethanol concentrations in adolescents rise more rapidly as compared to adults. According to the NIAAA (National Institute on Alcohol Abuse and Alcoholism), binge drinking in adults corresponds to 5 drinks within a 2 hour period, which lead to a blood concentration above $80 \mathrm{mg} / \mathrm{dl}$. In the adolescent population, three drinks only are sufficient to pass the threshold (Donovan, 2009), thus increasing the likelihood that a concentration close to that used in our experiments is reached. Therefore, our data contribute to clarify that even single, acute exposures to alcohol can have dramatic and potential long-term consequences on brain electrical activity and behavior. 


\section{References}

Atkinson SE, Williams SR (2009) Postnatal development of dendritic synaptic integration in rat neocortical pyramidal neurons. J Neurophysiol 102:735-751. doi: 10.1152/jn.00083.2009.

Badanich KA, Mulholland PJ, Beckley JT, Trantham-Davidson H, Woodward JJ (2013) Ethanol reduces neuronal excitability of lateral orbitofrontal cortex neurons via a glycine receptor dependent mechanism. Neuropsychopharmacology 38:1176-1188. doi: 10.1038/npp.2013.12.

Brodie MS, Appel SB (2000) Dopaminergic neurons in the ventral tegmental area of C57BL/6J and DBA/2J mice differ in sensitivity to ethanol excitation. Alcohol Clin Exp Res 24:1120-1124.

Chen Y, Wu P, Fan X, Chen H, Yang J, Song T, Huang C (2012) Ethanol enhances human hyperpolarization-activated cyclic nucleotide-gated currents. Alcohol Clin Exp Res 36:2036-2046. doi: 10.1111/j.1530-0277.2012.01826.x.

Cohen EJ, Quarta E, Bravi R, Granato A, Minciacchi D (2017) Neural plasticity and network remodeling: From concepts to pathology. Neuroscience. 344:326-345. doi: 10.1016/j.neuroscience.2016.12.048.

Criswell HE, Ming Z, Kelm MK, Breese GR (2008) Brain regional differences in the effect of ethanol on GABA release from presynaptic terminals. J Pharmacol Exp Ther. 326: 596-603. doi: 10.1124/jpet.107.135418.

Day M, Carr DB, Ulrich S, Ilijic E, Tkatch T, Surmeier DJ (2005) Dendritic excitability of mouse frontal cortex pyramidal neurons is shaped by the interaction among HCN, Kir2, and Kleak channels. J Neurosci 25: 8776-8787.

Donovan JE (2009) Estimated blood alcohol concentrations for child and adolescent drinking and their implications for screening instruments. Pediatrics. 123:e975-981. doi: 10.1542/peds.20080027.

Donovan JE (2013) The burden of alcohol use: focus on children and preadolescents. Alcohol Res 35:186-192.

Dou X, Wilkemeyer MF, Menkari CE, Parnell SE, Sulik KK, Charness ME (2013) Mitogenactivated protein kinase modulates ethanol inhibition of cell adhesion mediated by the L1 neural cell adhesion molecule. Proc Natl Acad Sci U S A 110: 5683-5688. doi: 10.1073/pnas.1221386110.

Ehrlich D, Pirchl M, Humpel C (2012) Ethanol transiently suppresses choline-acetyltransferase in basal nucleus of Meynert slices. Brain Res 1459: 35-42. doi: 10.1016/j.brainres.2012.04.020.

Fleming RL, Manis PB, Morrow AL (2009) The effects of acute and chronic ethanol exposure on presynaptic and postsynaptic gamma-aminobutyric acid (GABA) neurotransmission in cultured cortical and hippocampal neurons. Alcohol 43: 603-618. doi: 10.1016/j.alcohol.2009.10.006.

Förstera B, Castro PA, Moraga-Cid G, Aguayo LG (2016) Potentiation of Gamma Aminobutyric Acid Receptors (GABAAR) by Ethanol: How Are Inhibitory Receptors Affected? Front Cell Neurosci. 10: 114. doi: 10.3389/fncel.2016.00114. 
Fuenzalida M, Fernandez de Sevilla D, Buño W (2007) Changes of the EPSP waveform regulate the temporal window for spike-timing-dependent plasticity. J Neurosci 27:11940-11948.

Galindo R, Zamudio PA, Valenzuela CF (2005) Alcohol is a potent stimulant of immature neuronal networks: implications for fetal alcohol spectrum disorder. J Neurochem 94:1500-1511.

Gilpin NW, Smith AD, Cole M, Weiss F, Koob GF, Richardson HN (2009) Operant behavior and alcohol levels in blood and brain of alcohol-dependent rats. Alcohol Clin Exp Res 33:2113-2123. doi: 10.1111/j.1530-0277.2009.01051.x

Granato A, Palmer LM, De Giorgio A, Tavian D, Larkum ME (2012) Early exposure to alcohol leads to permanent impairment of dendritic excitability in neocortical pyramidal neurons. $\mathrm{J}$ Neurosci 32:1377-1382. doi: 10.1523/JNEUROSCI.5520-11.2012.

Han CL, Liao CS, Wu CW, Hwong CL, Lee AR, Yin SJ (1998) Contribution to first-pass metabolism of ethanol and inhibition by ethanol for retinol oxidation in human alcohol dehydrogenase family--implications for etiology of fetal alcohol syndrome and alcohol-related diseases. Eur J Biochem 254: 25-31.

Heit C, Dong H, Chen Y, Shah YM, Thompson DC, Vasiliou V (2015) Transgenic mouse models for alcohol metabolism, toxicity, and cancer. Adv Exp Med Biol 815:375-87. doi: 10.1007/978-3319-09614-8_22.

Hingson RW, Heeren T, Winter MR (2006) Age at drinking onset and alcohol dependence: age at onset, duration, and severity. Arch Pediatr Adolesc Med 160:739-746.

Huang JJ, Yen CT, Tsai ML, Valenzuela CF, Huang C (2012) Acute ethanol exposure increases firing and induces oscillations in cerebellar Golgi cells of freely moving rats. Alcohol Clin Exp Res 36:2110-2116. doi: 10.1111/j.1530-0277.2012.01818.x.

Ikonomidou C, Bittigau P, Ishimaru MJ, Wozniak DF, Koch C, Genz K, Price MT, Stefovska V, Hörster F, Tenkova T, Dikranian K, Olney JW (2000) Ethanol-induced apoptotic neurodegeneration and fetal alcohol syndrome. Science 287: 1056-1060.

Lobo IA, Harris RA (2008) GABA(A) receptors and alcohol. Pharmacol Biochem Behav. 90:90-94. doi: $10.1016 /$ j.pbb.2008.03.006.

Lotfullina N, Khazipov R (2017) Ethanol and the Developing Brain: Inhibition of Neuronal Activity and Neuroapoptosis. Neuroscientist. doi: 10.1177/1073858417712667.

Marszalec W, Aistrup GL, Narahashi T (1998) Ethanol modulation of excitatory and inhibitory synaptic interactions in cultured cortical neurons. Alcohol Clin Exp Res. 22:1516-1524.

McMurray MS, Amodeo LR, Roitman JD (2016) Consequences of Adolescent Ethanol Consumption on Risk Preference and Orbitofrontal Cortex Encoding of Reward. Neuropsychopharmacology 41:1366-1375. doi: 10.1038/npp.2015.288.

Moriguchi S, Zhao X, Marszalec W, Yeh JZ, Narahashi T (2007) Effects of ethanol on excitatory and inhibitory synaptic transmission in rat cortical neurons. Alcohol Clin Exp Res 31:89-99. 
Okamoto T, Harnett MT, Morikawa H (2006) Hyperpolarization-activated cation current (Ih) is an ethanol target in midbrain dopamine neurons of mice. J Neurophysiol 95:619-626.

Olney JW (2014) Focus on apoptosis to decipher how alcohol and many other drugs disrupt brain development. Front Pediatr 2:81. doi: 10.3389/fped.2014.00081.

Proctor WR, Soldo BL, Allan AM, Dunwiddie TV (1992) Ethanol enhances synaptically evoked GABAA receptor-mediated responses in cerebral cortical neurons in rat brain slices. Brain Res 595:220-227.

Ramaswamy S, Markram H (2015) Anatomy and physiology of the thick-tufted layer 5 pyramidal neuron. Front Cell Neurosci 9:233. doi: 10.3389/fncel.2015.00233

Rehm J, Mathers C, Popova S, Thavorncharoensap M, Teerawattananon Y, Patra J (2009) Global burden of disease and injury and economic cost attributable to alcohol use and alcohol-use disorders. Lancet. 373:2223-2233. doi: 10.1016/S0140-6736(09)60746-7.

Sanderson JL, Donald Partridge L, Valenzuela CF (2009) Modulation of GABAergic and glutamatergic transmission by ethanol in the developing neocortex: an in vitro test of the excessive inhibition hypothesis of fetal alcohol spectrum disorder. Neuropharmacology 56:541-555. doi: 10.1016/j.neuropharm.2008.10.012

Sessler FM, Hsu FC, Felder TN, Zhai J, Lin RC, Wieland SJ, Kosobud AE (1998) Effects of ethanol on rat somatosensory cortical neurons. Brain Res 804:266-274.

Siggins GR, Pittman QJ, French ED (1987) Effects of ethanol on CA1 and CA3 pyramidal cells in the hippocampal slice preparation: an intracellular study. Brain Res 414:22-34.

Smolen TN, Smolen A (1989) Blood and brain ethanol concentrations during absorption and distribution in long-sleep and short-sleep mice. Alcohol 6:33-38.

Spear LP (2016) Consequences of adolescent use of alcohol and other drugs: Studies using rodent models. Neurosci Biobehav Rev 70: 228-243. doi: 10.1016/j.neubiorev.2016.07.026.

Tipps ME, Raybuck JD, Lattal KM (2014). Substance abuse, memory, and post-traumatic stress disorder. Neurobiol Learn Mem 112:87-100. doi: 10.1016/j.nlm.2013.12.002.

Trudell JR, Messing RO, Mayfield J, Harris RA (2014) Alcohol dependence: molecular and behavioral evidence. Trends Pharmacol Sci 35:317-3723. doi: 10.1016/j.tips.2014.04.009.

Wei W, Faria LC, Mody I (2004) Low ethanol concentrations selectively augment the tonic inhibition mediated by delta subunit-containing GABAA receptors in hippocampal neurons. $\mathbf{J}$ Neurosci 24:8379-8382.

Weiner JL, Valenzuela CF (2006) Ethanol modulation of GABAergic transmission: the view from the slice. Pharmacol Ther 111:533-354.

White AM (2003) What happened? Alcohol, memory blackouts, and the brain. Alcohol Res Health 27:186-196. 
Yan H, Li Q, Fleming R, Madison RD, Wilson WA, Swartzwelder HS (2009) Developmental sensitivity of hippocampal interneurons to ethanol: involvement of the hyperpolarization-activated current, Ih. J Neurophysiol 101:67-83. doi: 10.1152/jn.90557.2008.

Zorumski CF, Mennerick S, Izumi Y (2014) Acute and chronic effects of ethanol on learningrelated synaptic plasticity. Alcohol 48:1-17. doi: 10.1016/j.alcohol.2013.09.045. 


\section{Figure legends}

Fig. 1. Staining of dead cells in mouse cortical slices after propidium iodide uptake. a-c: representative microscopy fields showing the incidence of dead cells in control slices (a) and slices treated with ethanol for 3 or $6 \mathrm{~min}(\mathbf{b}, \mathbf{c})$. Note that the nuclei of dead cells are intensely fluorescent and stand out very neatly over tissue background. Scale bar $=100 \mu \mathrm{m}$. d: graph showing the result of quantitative analysis of the density (positive nuclei $/ \mathrm{mm}^{2}$ ) of dead cells in control and ethanol-treated slices.

Fig. 2 a-d. Subthreshold parameters measured before ethanol exposure (preEt), 3 minutes, and 6 minutes after the beginning of ethanol superfusion $(3 \mathrm{mEt}, 6 \mathrm{mEt})$. RMP: resting membrane potential; $\mathrm{n}=8$ cells. $*: \mathrm{P}<0.05$. ${ }^{* *}: \mathrm{P}<0.01$. Broken lines beside each graph show the values recorded from single neurons for each experimental condition.

Fig. 3 The main firing properties measured before ethanol exposure (preEt), 3 minutes, and 6 minutes after the beginning of alcohol superfusion $(3 \mathrm{mEt}, 6 \mathrm{mEt})$. a: representative recordings from the same L5 pyramidal neuron. For all the recordings, both the hyperpolarizing and the depolarizing current were $100 \mathrm{pA}$. b-d: mean interspike interval (ISI) at rheobase, $50 \mathrm{pA}$, and $100 \mathrm{pA}$ above rheobase (where rheobase is that found for each neuron before ethanol application). The symbols (x) indicate the number of neurons with less than two spikes / train. In these cases the ISI was approximated to $801 \mathrm{~ms}$ ( 0 spike / train) or to the longest interval between the single spike and the extreme points of the depolarization envelope. The broken lines beside $\mathrm{b}$ show the ISI recorded from single neurons at rheobase, for each experimental condition. The symbols (\#) indicate recordings with 0 spike / train in two neurons. Inset of c: ratio between the last and the first ISI of the spike train $50 \mathrm{pA}$ above rheobase. e-h: the main parameters regarding the action potentials. $\mathrm{AHP}=$ afterhyperpolarization; $\mathrm{n}=8$ cells. $*: \mathrm{P}<0.05$. **: $\mathrm{P}<0.01$

Fig. 4 Effects of ethanol on mIPSCs. a: Representative voltage clamp recordings of mIPSCs from a L5 pyramidal neuron before ethanol exposure (preEt), and after $3(3 \mathrm{mEt})$ and 6 minutes $(6 \mathrm{mEt})$ of exposure. b-c: Pooled data of mIPSC frequency $(n=4)$ and amplitude $(n=4)$. d: Averaged mIPSCs obtained at preEt and at $6 \mathrm{mEt}$ from the neuron in $\mathbf{a}$ and scaled at the peak amplitude for comparing time courses. e-f Pooled data of mIPSC rise time $(n=4)$ and decay time $(n=4)$. Broken lines beside $b$-c and e-f show the values recorded from single neurons for each experimental condition *: $\mathrm{P}<0.05$. 

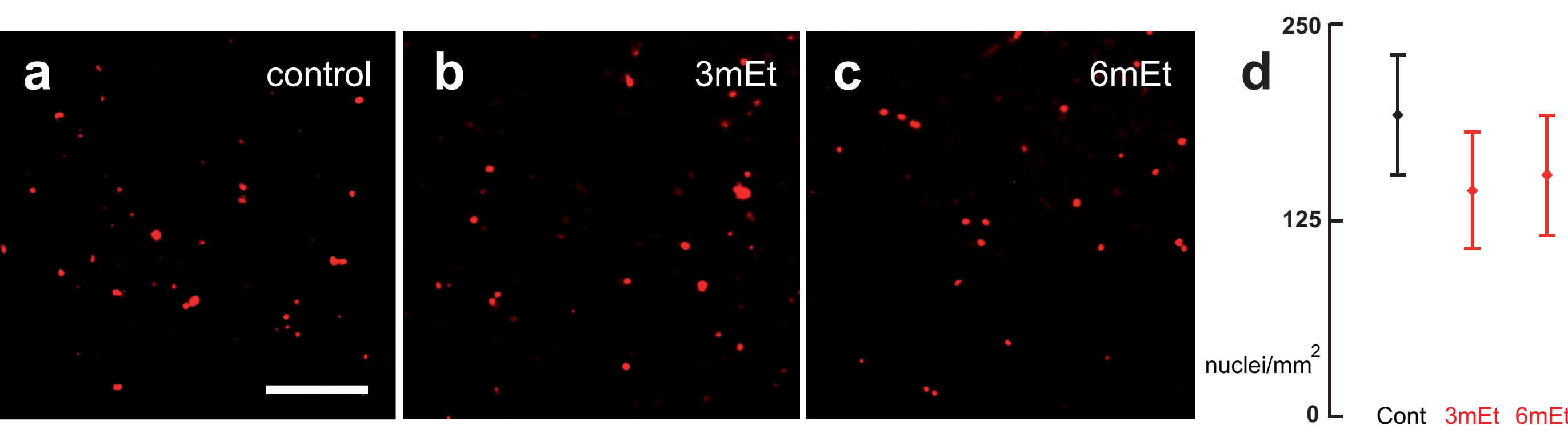
Click here to ${ }^{3}$ ddwwnload Figure Figure2.eps $\underline{\underline{ \pm}}$

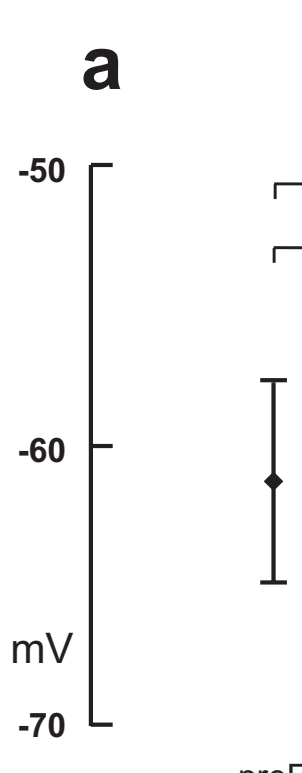

preEt
RMP
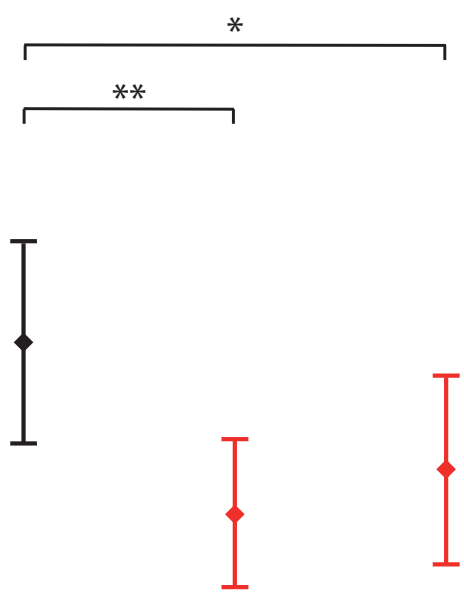

$3 \mathrm{mEt}$
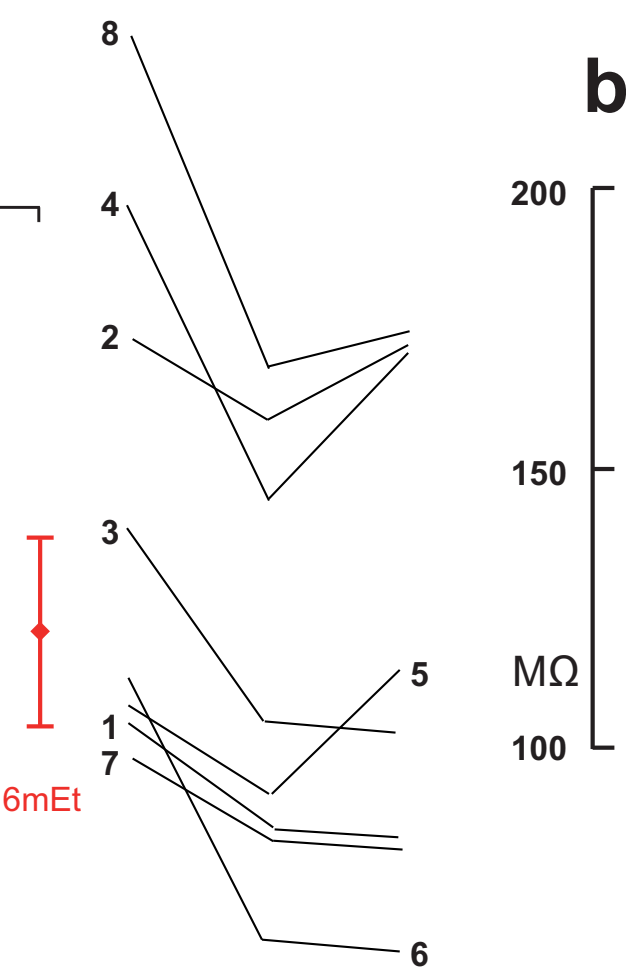

b

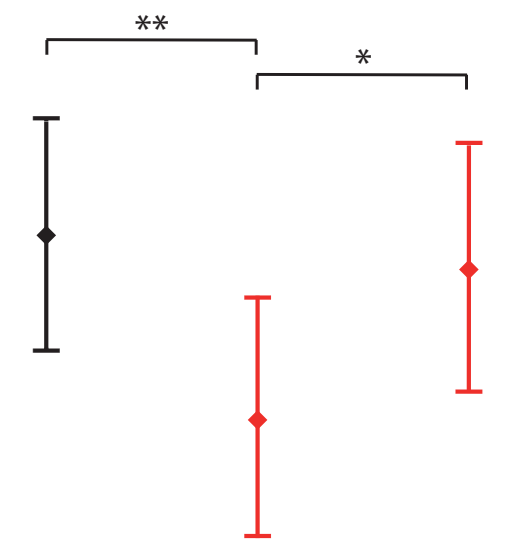

preEt

$3 \mathrm{mEt}$

$6 \mathrm{mEt}$

preEt

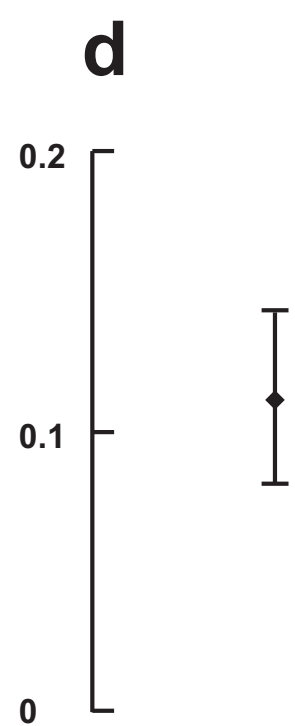

Sag

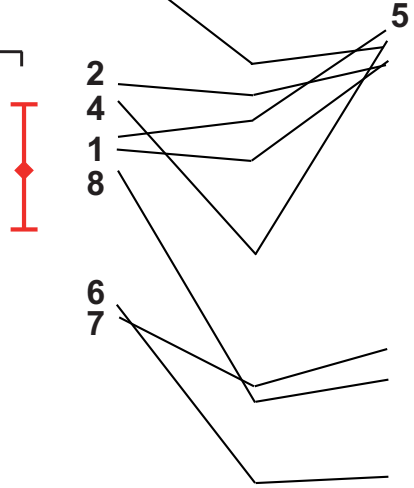

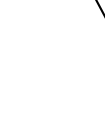

1
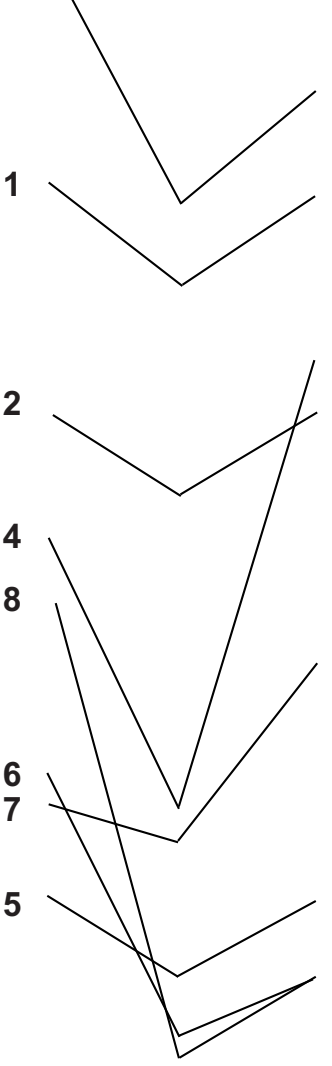

1

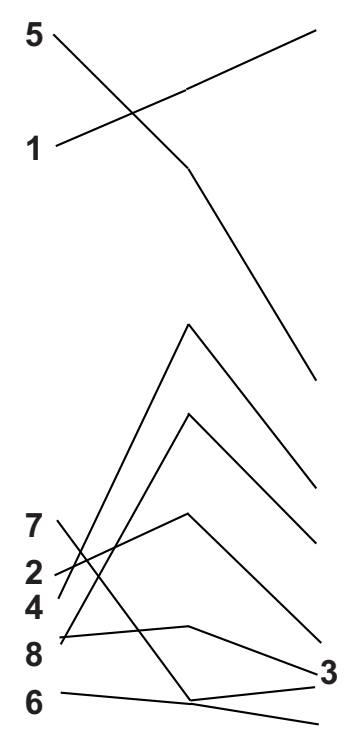



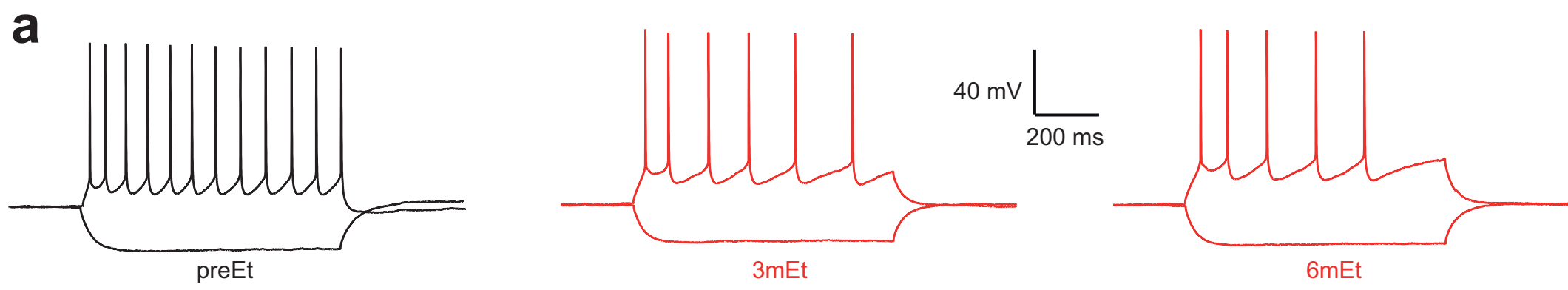
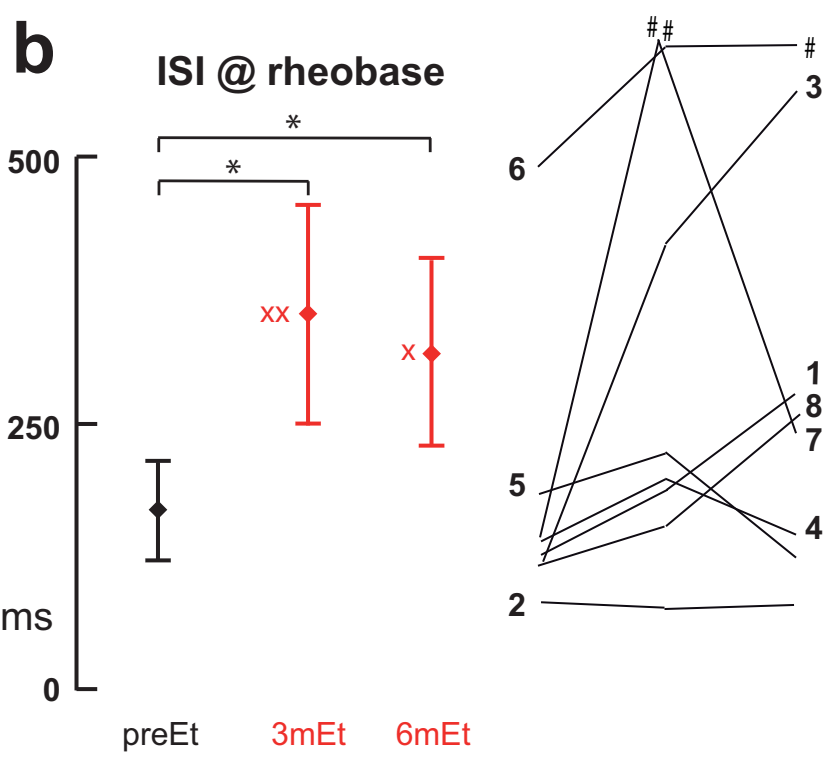

2
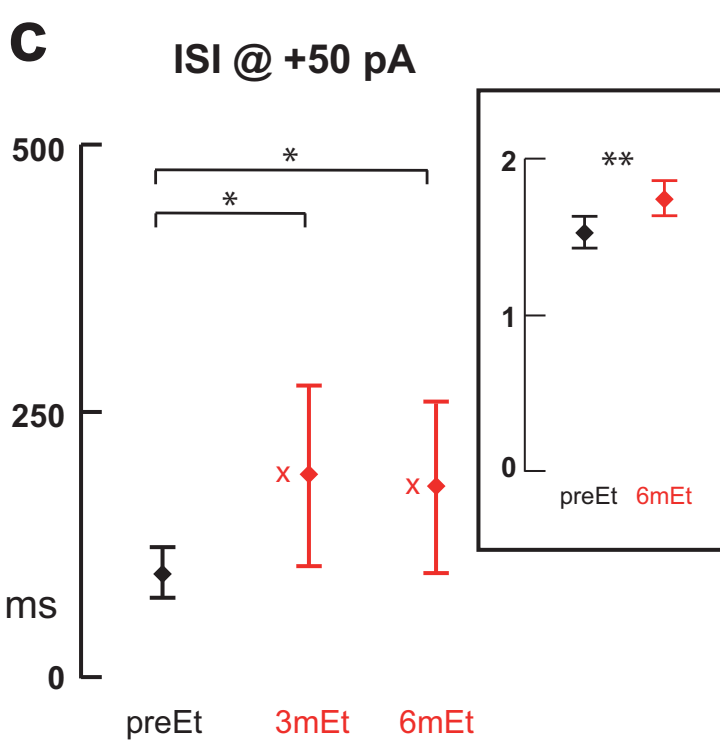

$\epsilon$

Threshold

$$
\begin{aligned}
& -2 \\
& -3 ! \\
& m \\
& -5 !
\end{aligned}
$$

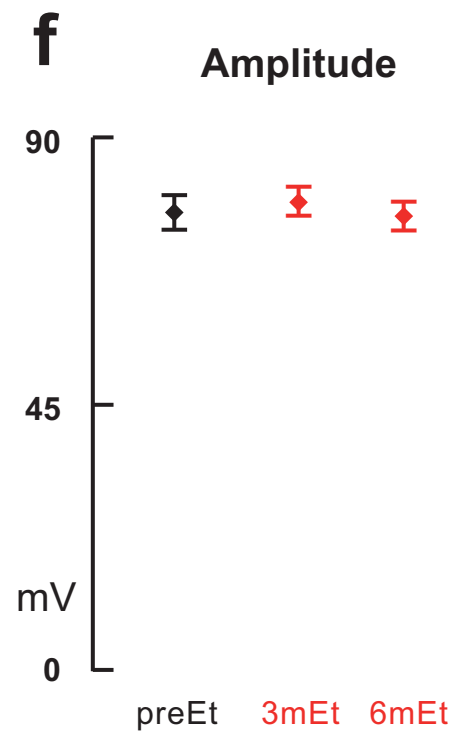

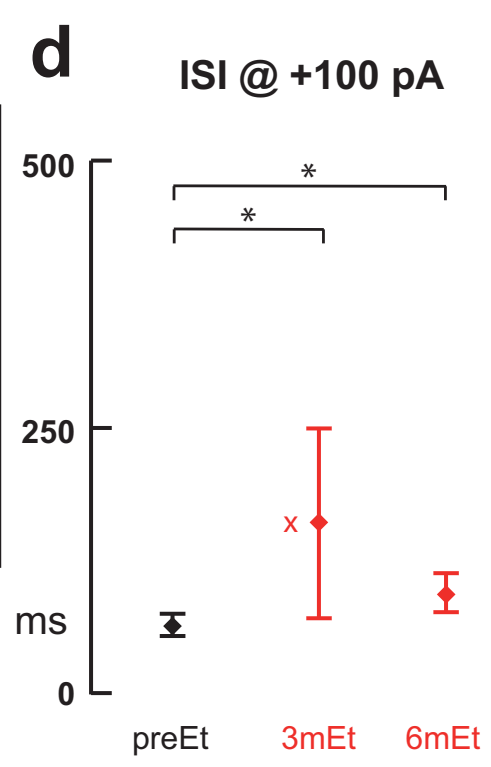

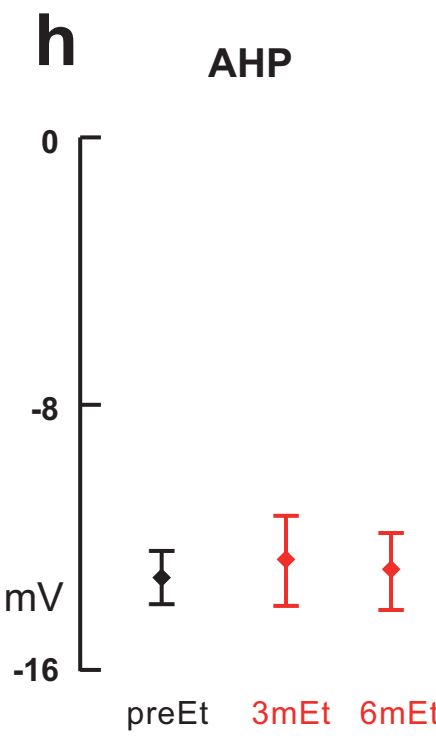


a

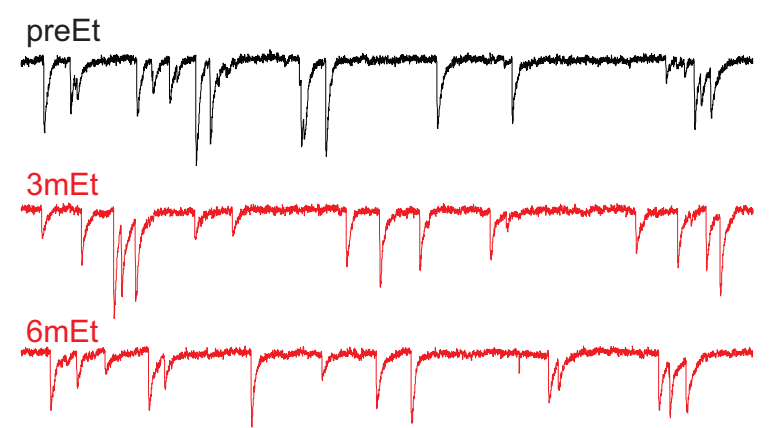

d

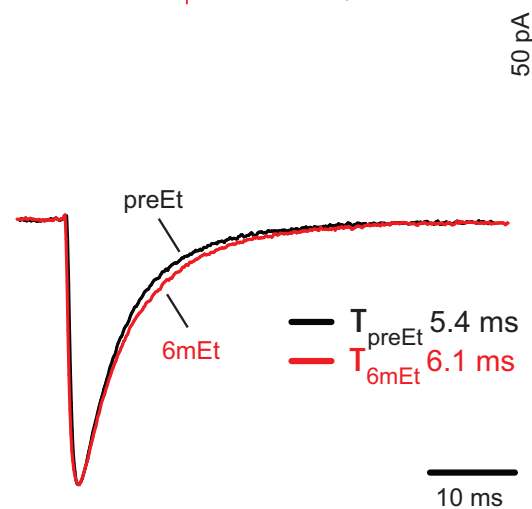

喆 b

Frequency
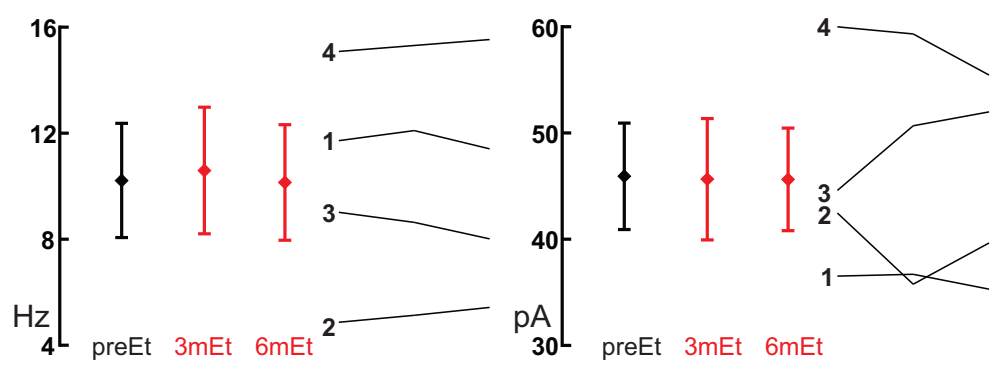

e

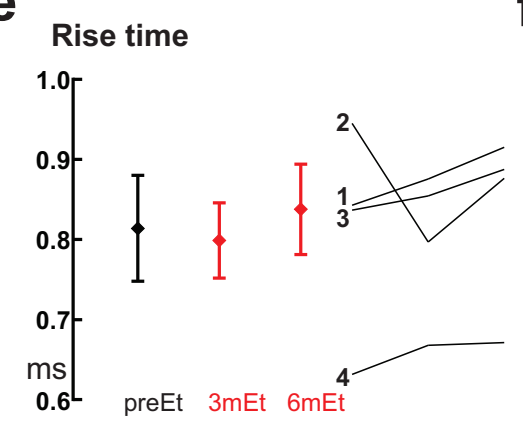

C

Amplitude

f Decay time

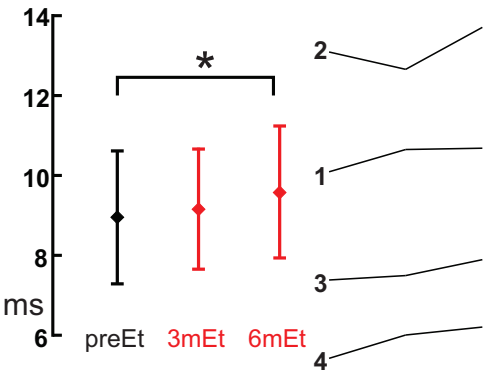

